\title{
Variable-Temperature Tip-Enhanced Raman Spectroscopy of Single- Molecule Fluctuations and Dynamics
}

\author{
Kyoung-Duck Park, ${ }^{\dagger}$ Eric A. Muller, ${ }^{\dagger}$ Vasily Kravtsov, ${ }^{\dagger}$ Paul M. Sass, ${ }^{\dagger}$ Jens Dreyer, $^{\dagger}$ Joanna M. Atkin, ${ }^{\dagger, \mathbb{I}}$
} and Markus B. Raschke*, ${ }^{*}$

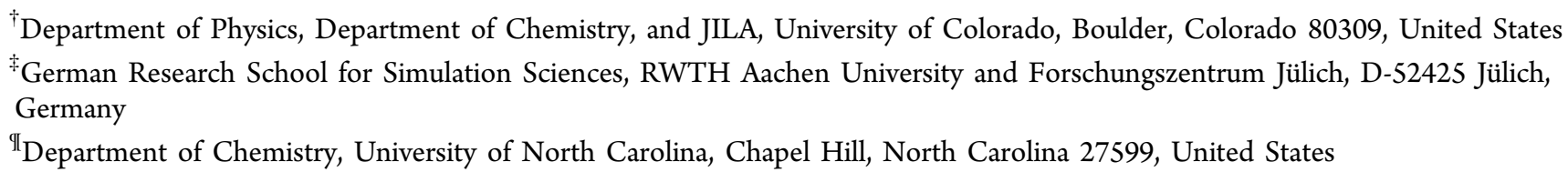

Supporting Information

ABSTRACT: Structure, dynamics, and coupling involving single-molecules determine function in catalytic, electronic or biological systems. While vibrational spectroscopy provides insight into molecular structure, rapid fluctuations blur the molecular trajectory even in single-molecule spectroscopy, analogous to spatial averaging in measuring large ensembles. To gain insight into intramolecular coupling, substrate coupling, and dynamic processes, we use tip-enhanced Raman spectroscopy (TERS) at variable and cryogenic temperatures, to slow and control the motion of a single molecule. We resolve intrinsic line widths of individual normal

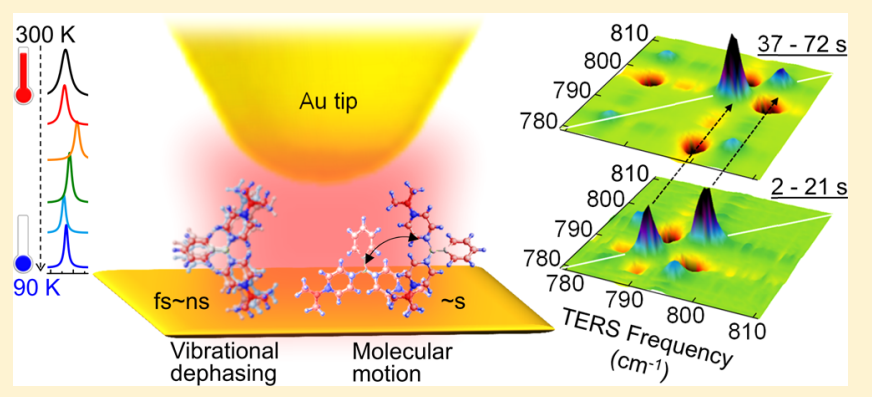
modes, allowing detailed and quantitative investigation of the vibrational modes. From temperature dependent line narrowing and splitting, we quantify ultrafast vibrational dephasing, intramolecular coupling, and conformational heterogeneity. Through statistical correlation analysis of fluctuations of individual modes, we observe rotational motion and spectral fluctuations of the molecule. This work demonstrates single-molecule vibrational spectroscopy beyond chemical identification, opening the possibility for a complete picture of molecular motion ranging from femtoseconds to minutes.

KEYWORDS: Tip-enhanced Raman spectroscopy (TERS), low-temperature, single molecule, molecular orientation, molecular dynamics, vibrational dephasing

$T^{1}$ he investigation of single molecules allows for the observation of unsynchronized processes and rare events occurring at specific locations in an inhomogeneous sample. As such, single-molecule spectroscopy provides the systematic basis to reach beyond traditional spectroscopies that average over both the spatial distribution and temporal dynamics of molecules.

Single-molecule spectroscopy can sensitively probe both local environment and dynamics through spatially- and time-varying shifts in fluorescence energies. ${ }^{1-4}$ Imaging the spatial distribution of an optical excitation, ${ }^{5}$ disorder in molecular crystals, ${ }^{6}$ or orientational fluctuations of a biomolecule ${ }^{7}$ gives insight into electronic and molecular dynamics unresolved in a bulk measurement.

However, single-molecule fluorescence and its variations only indirectly probe the effects of local perturbations such as external fields, intermolecular coupling, conformational states, or strain and are generally not structurally specific. $^{8-10}$ Similarly, multi-dimensional spectroscopy provides insight into intramolecular structure and dynamics on ultrafast time scales, yet with limited information about slower time scales, oscillator density, or real-space distribution. ${ }^{11}$
Vibrational spectroscopy can directly probe molecular identity through characteristic spectral "fingerprints". Vibrational resonances can also serve as exquisitely sensitive and specific reporters of intramolecular dynamics and the local molecular environment due to the structural specificity of normal modes and their narrow line width. Despite the relatively small cross section typically associated with Raman scattering, enhancement by electronic resonances and metal plasmons have enabled single-molecule vibrational spectroscopy with surface-enhanced Raman scattering (SERS) ${ }^{12,13}$ and tip-enhanced Raman spectroscopy (TERS). ${ }^{14,15}$ The high sensitivity and spatial resolution of TERS enables investigation of localized chemical features and processes even in a dense and heterogeneous system. ${ }^{16,17}$

However, molecules may be highly mobile at room temperature, and single-molecule TERS or SERS spectra average over many adsorbate conformations. Rapid spectral fluctuations that occur faster than the spectral acquisition time

Received: October 10, 2015

Revised: November 30, 2015

Published: December 17, 2015 
(a)

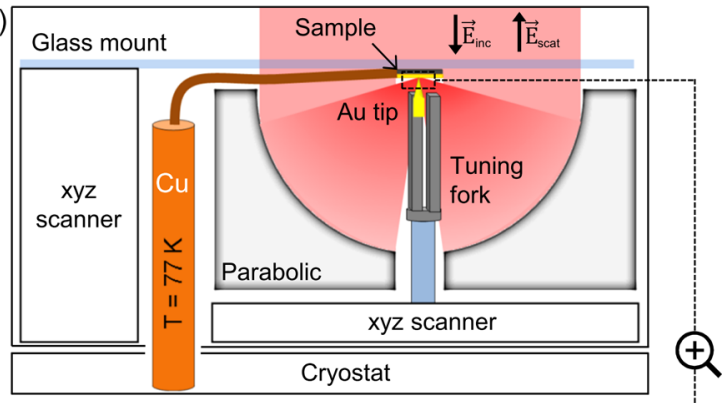

(b)

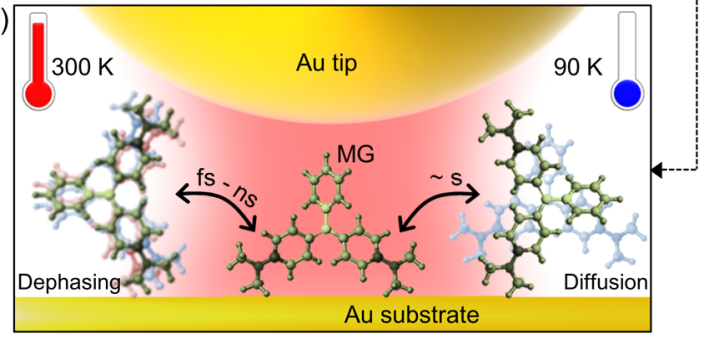

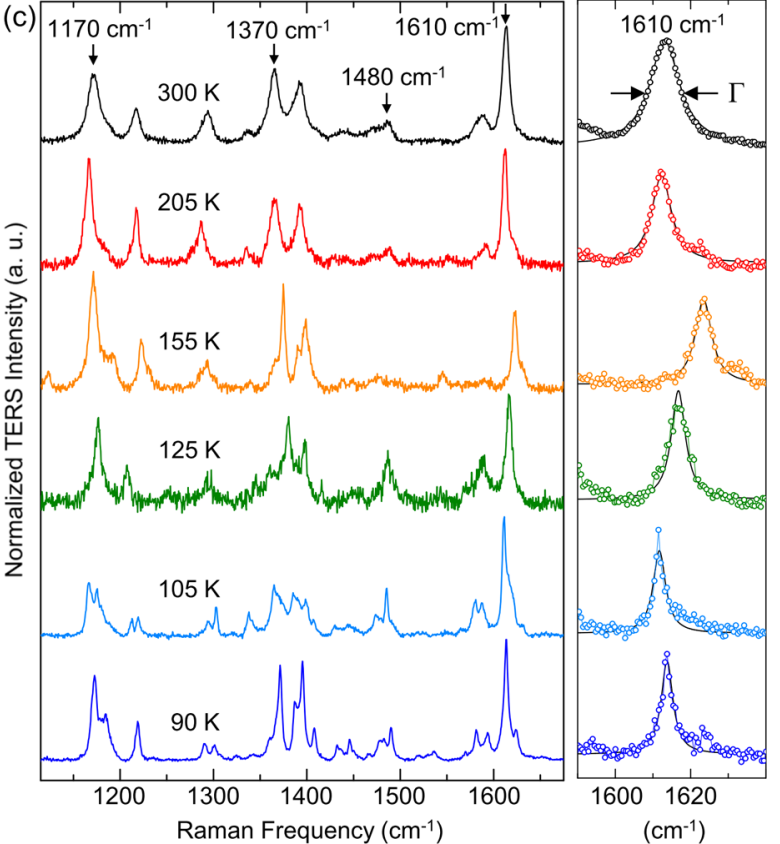

Figure 1. (a) Schematic of the cryogenic TERS experiment. Incident laser $\left(E_{\text {inc }}\right)$ is focused onto the apex of an etched Au AFM tip and Raman signal $\left(E_{\text {scat }}\right)$ is collected by a parabolic mirror in a back scattering geometry. (b) Illustration of the tip-substrate junction showing molecular motion of malachite green (MG) with characteristic vibrational signatures at different temperatures. (c) Temperature-dependent TERS spectra of MG. Spectra are background subtracted and intensity normalized. Lorentzian line fit analysis of the $\mathrm{N}-\mathrm{C}$ stretch mode at $1610 \mathrm{~cm}^{-1}$ as an example (Right graph of (c), narrowest spectra are selected (acquisition times: 1-10 s) from a large data set, additional fits shown in Supporting Information Figure S2b).

contribute to broadening, similar to the ensemble averaged spectra in far-field spectroscopy. ${ }^{12,18}$ Recently, low-temperature TERS has allowed for the spectroscopy of single molecules with static translational and orientational conformation and the intrinsic line width yet unresolved. ${ }^{19,20}$ Temperature-dependent line shape measurements in SERS suggest the possibility to distinguish vibrational dephasing on the femtosecond time scale. ${ }^{21}$ Low- and variable-temperature TERS would allow for the selective probing of single-molecule intramolecular vibrational coupling. However, other contributions to the spectrum from orientational motion and spectral fluctuations may also contribute to spectral line shapes in TERS even in the limit of single molecules.

Here, we perform cryogenic and variable temperature (90$300 \mathrm{~K})$ TERS of malachite green (MG) with high spectral resolution. In order to understand the role of intramolecular coupling, conformational heterogeneity, dephasing, and ensemble effects, we perform temperature-dependent TERS to observe thermally activated broadening. At low temperatures, we observe intensity fluctuations on the seconds time scale that are both correlated and anticorrelated between modes, as well as frequency fluctuations greater than the intrinsic line width. We attribute these fluctuations to rotational motion and dynamic changes in the local environment of a single molecule. We demonstrate that at the small-ensemble or single-molecule limit, temperature-dependent line shape and time-dependent spectral fluctuations are intrinsically linked through multitimescale dynamic interactions.

Results. The TERS experiments are performed, using electrochemically etched $\mathrm{Au}$ tips with $\sim 10 \mathrm{~nm}$ apex radius, with high spectral resolution $\left(\sim 1.2 \mathrm{~cm}^{-1}\right)$, in a high vacuum environment as shown in Figure la,b. A sparse submonolayer MG coverage adsorbed on a flat template stripped Au substrate enables investigation of small clusters and single-molecules. We compare TERS with far-field Raman spectroscopy (microRaman) at each temperature. For a complete description of the experiment see Methods and Supporting Information.

Figure 1c shows TERS spectra of submonolayer MG in the frequency range $1125-1675 \mathrm{~cm}^{-1}$ (full spectrum in Supporting Information Figure S2a) acquired at different temperatures. At $300 \mathrm{~K}$, we observe broad peaks with $10-20 \mathrm{~cm}^{-1}$ full width at half-maximum (FWHM), which is in agreement with previous room-temperature TERS. ${ }^{22}$ These peaks result from the spectrally unresolved superposition of inhomogeneously and thermally broadened Raman active vibrational modes. The signal emerges from the only a few molecules within the nanoscale sample volume (average of $\sim 1-2$ molecules) as calculated from the near-field spatial localization and surface MG density. The TERS line widths are systematically narrower than the ensemble measurements using micro-Raman. The micro-Raman ensemble measurement of a heterogeneous system shows significantly broader line shapes at all temperatures due to inhomogeneous broadening from the ensemble average over $\sim 4 \times 10^{5}$ molecules. In contrast, TERS spectra only contain inhomogeneous broadening contributions from the range of conformations and adsorption sites sampled by a small ensemble of molecules. ${ }^{23}$

Shown in Figure 1c, the initially broad peaks narrow with decreasing temperature, and several of them split, revealing a number of additional modes. At $90 \mathrm{~K}$, narrow line widths allow for the full identification of the normal modes and symmetries of each TERS peak (see Supporting Information Figure S2a), otherwise obscured at room temperature. Within the spectral range shown, we assign selected peaks of characteristic normal modes based on density functional theory (DFT) calculations, including $\mathrm{C}-\mathrm{H}$ in-plane bends $\left(1170 \mathrm{~cm}^{-1}\right)$ and twists $(1370$ $\left.\mathrm{cm}^{-1}\right)$, bending of the methyl group $\left(1480 \mathrm{~cm}^{-1}\right)$, and an $\mathrm{N}-\mathrm{C}$ stretching mode $\left(1610 \mathrm{~cm}^{-1}\right)$. 
In order to gain insight into the underlying mechanisms, we analyze the temperature dependence of vibrational line widths $\Gamma(T)$ and resonance frequencies $\bar{\nu}(T)$. Figure 2 shows the

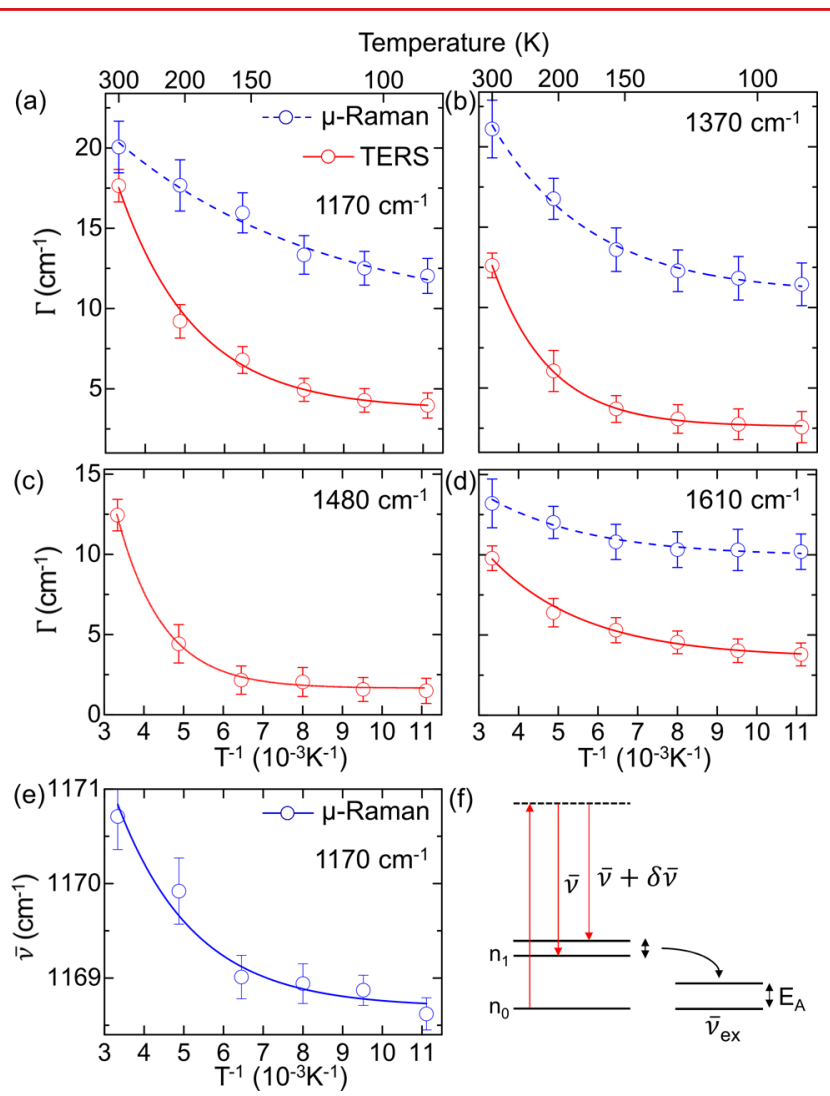

Figure 2. Temperature-dependent TERS and micro-Raman line widths $\Gamma(T)$ (a-d), and micro-Raman center frequency $\bar{\nu}(T)$ (e) with corresponding exponential fits. (f) Energy diagram of an energy exchange model for vibrational dephasing. A vibrational mode with ground state $n_{0}$ and excited state $n_{1}$ is coupled to an exchange mode $\bar{\nu}_{\text {ex }}$. Dephasing is caused by instantaneous changes $\delta \bar{\nu}$ of the vibrational Raman frequency $\bar{\nu}$.

results for the four selected modes both from TERS and microRaman spectroscopy. The line widths $\Gamma(T)$ and uncertainties have been extracted from a Lorentzian line fit analysis (shown in the right graph of Figure 1c and Supporting Information Figure S2b). Because the narrowest peaks are selected from the fluctuating spectra in the time domain, we can select a homogeneous subensemble and thus eliminate heterogeneity otherwise observed in an ensemble measurement. ${ }^{24}$

At room temperature, TERS line widths are $2-8 \mathrm{~cm}^{-1}$ narrower compared to the line width obtained with microRaman, while at $90 \mathrm{~K}$, TERS line widths are $6-10 \mathrm{~cm}^{-1}$ less than micro-Raman. As an example, the micro-Raman line widths of the $1170 \mathrm{~cm}^{-1} \mathrm{C}-\mathrm{H}$ bend narrows only by a factor of 2, from $20 \mathrm{~cm}^{-1}$ at $300 \mathrm{~K}$ to $12 \mathrm{~cm}^{-1}$ at $90 \mathrm{~K}$ (Figure $2 \mathrm{a}$ ). In contrast, the same $1170 \mathrm{~cm}^{-1}$ mode measured by TERS narrows by a factor of $\sim 5$ over the same temperature range, from 18 to $3.5 \mathrm{~cm}^{-1}$.

Temperature dependence of the line widths is found to follow an Arrhenius behavior. Figure $2 \mathrm{a}-\mathrm{d}$ shows fits for $\Gamma(T)$ $=\Gamma_{0}+A \cdot e^{-E_{A} / k_{\mathrm{b}} T}$. Fit parameters for activation energy $E_{\mathrm{A}}$, zero temperature line width $\Gamma_{0}$, and prefactor $A$ from TERS are summarized in Table 1 (the corresponding micro-Raman results can be found in Supporting InformationTable S1). The
Table 1. Results from Arrhenius Fits to the Temperature Dependent TERS Line Width $\Gamma(T)^{a}$

$\begin{array}{cccc}\text { peak }\left(\mathrm{cm}^{-1}\right) & E_{\mathrm{A}}\left(\mathrm{cm}^{-1}\right) & \Gamma_{0}\left(\mathrm{~cm}^{-1}\right) & A\left(\mathrm{~cm}^{-1}\right) \\ \bar{\nu}(1610) & 290 \pm 40 & 3.5 \pm 0.4 & 25 \pm 6 \\ \bar{\nu}(1480) & 590 \pm 60 & 1.5 \pm 0.2 & 180 \pm 40 \\ \bar{\nu}(1370) & 480 \pm 20 & 2.6 \pm 0.2 & 98 \pm 8 \\ \bar{\nu}(1170) & 360 \pm 40 & 3.7 \pm 0.6 & 77 \pm 20 \\ \text { peak }\left(\mathrm{cm}^{-1}\right) & E_{\mathrm{A}}\left(\mathrm{cm}^{-1}\right) & \bar{\nu}_{0}\left(\mathrm{~cm}^{-1}\right) & A\left(\mathrm{~cm}^{-1}\right) \\ \bar{\nu}_{F}(1170) & 270 \pm 80 & 1168.5 \pm 0.2 & 10 \pm 4\end{array}$

${ }^{a}$ Four selected peaks over the temperature range $90-300 \mathrm{~K}$ (top) and corresponding fits to the variation in center frequency $\bar{\nu}_{F}(T)$ measured by micro-Raman spectroscopy for the $1170 \mathrm{~cm}^{-1}$ mode (bottom).

fitted values obtained for $E_{\mathrm{A}}\left(290-590 \mathrm{~cm}^{-1}\right)$ and $A(25-180$ $\mathrm{cm}^{-1}$ ) in TERS are larger than micro-Raman, as discussed in detail below.

Accompanying the decreasing line widths, the center frequencies of several modes are found to continuously red shift with decreasing temperature in micro-Raman. As an example, Figure 2e shows the temperature dependence of the micro-Raman center frequency $\bar{\nu}(T)$ of the $1170 \mathrm{~cm}^{-1}$ mode with $E_{\mathrm{A}}=270 \pm 80 \mathrm{~cm}^{-1}$. However, as seen in Figure 1c, center frequencies in TERS typically do not shift in a systematic fashion at low temperatures. While TERS spectra at room temperature are reproducible across the sample and in repeated measurements, low-temperature TERS spectra do not have a reproducible center frequency due to inhomogeneity between small subensembles.

Following established procedures derived from early dephasing studies in vibrational spectroscopy, we model $\Gamma(T)$ and frequency shift $\Delta \bar{\nu}(T)$ as vibrational dephasing resulting from coupling $\delta \bar{\nu}$ to the thermal bath. ${ }^{25}$ An exchange coupling model, illustrated in Figure $2 \mathrm{f}$, approximates system-bath coupling as mediated primarily through a low energy vibrational exchange mode expressed as $\bar{\nu}_{\mathrm{ex}}{ }^{26-29}$ In the perturbative coupling regime, $\Gamma(T)$ and $\Delta \bar{\nu}(T)$ can be expressed as ${ }^{26}$

$$
\Gamma(T)=\Gamma_{0}+\frac{(\delta \bar{\nu})^{2} \tau}{1+(\delta \bar{\nu})^{2} \tau^{2}} \exp \left(-\frac{E_{\mathrm{A}}}{k T}\right)
$$

and

$$
\Delta \bar{\nu}(T)=\frac{\delta \bar{\nu}}{1+(\delta \bar{\nu})^{2} \tau^{2}} \exp \left(-\frac{E_{\mathrm{A}}}{k T}\right)
$$

An Arrhenius temperature dependence arises from the energy $E_{\mathrm{A}}$ associated with the vibrational exchange mode $\bar{\nu}_{\mathrm{ex}}$. The observed $\Gamma(T)$ and $\Delta \bar{\nu}(T)$ depend on the exchange coupling strength $\delta \bar{\nu}$ and the lifetime $\tau$ of the exchange mode. TERS measurements of $\bar{\nu}(T)$ shift randomly for each sub-ensemble measurement at lower temperatures, and $\Delta \bar{\nu}(T)$ is measured using micro-Raman following established procedures. ${ }^{21}$ From the combination of eqs 1 and $2, \delta \bar{\nu}$ and $\tau$ can be estimated. ${ }^{30}$ For the $1170 \mathrm{~cm}^{-1}$ mode, we find $\delta \bar{\nu}=110 \pm 50 \mathrm{~cm}^{-1}$ and $\tau=$ $80 \pm 20$ fs.

TERS spectra at low temperatures exhibit fluctuations in both intensity and frequency of individual peaks between subsequent measurements or between subsequent spectra in time-series acquisitions. Shown in Figure 3a, we collect time series spectra with high spectral resolution and acquisition times of $1 \mathrm{~s}$ (a longer time series is shown in Supporting Information Figure S4a). The high vacuum and low temperature of $90 \mathrm{~K}$ allows for measurement times of several minutes 

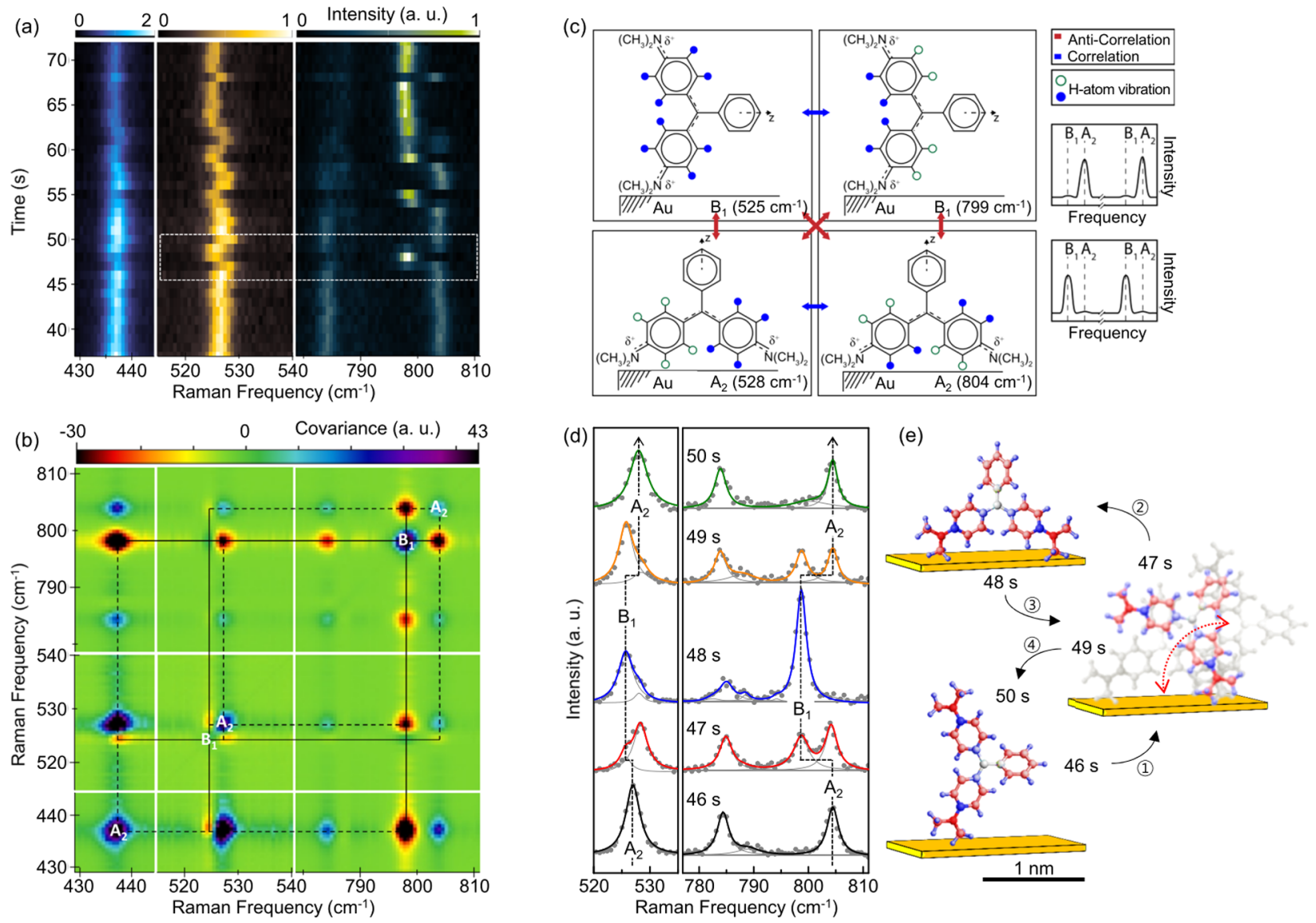

Figure 3. (a) Time-series contour plot of $90 \mathrm{~K}$ TERS spectra showing spectral fluctuations (for full spectral and temporal data set, see Supporting Information Figure S4a). (b) Corresponding covariance map of Raman intensities from (a). (c) Symmetry assignment of the four principle vibrational modes with the molecule shown in the orientation in which $A_{2}$ or $B_{1}$ modes are forbidden, respectively. (Anti) correlations from (b) are indicated by (red) blue arrows. The $\mathrm{C}-\mathrm{H}$ out-of-plane bending modes are highlighted by green and blue circles. (d) Subset of time-series spectra (dots) from the region indicated by the dashed box in (a) with Lorentzian fits (solid) exhibit alternating appearance of peaks at 525 and $799 \mathrm{~cm}^{-1}$ $\left(B_{1}\right)$ versus 528 and $804 \mathrm{~cm}^{-1}\left(A_{2}\right)$. (e) Corresponding molecular orientations at each time slice determined by mode symmetry.

without bleaching. While some peaks maintain constant frequency such as the $580 \mathrm{~cm}^{-1}$ mode (Supporting Information Figure S5a), several other peaks near 525 and $800 \mathrm{~cm}^{-1}$ fluctuate between discrete spectral positions.

From the full data set (Supporting Information Figure S4a), we calculate the covariance $\sigma(i, j)$ between frequencies $i$ and $j$ from the TERS intensities $I(i, t)$ during short time segments $t_{1}$ to $t_{2}$ using

$$
\sigma(i, j)=\frac{1}{N} \sum_{t=t_{1}}^{t_{2}}[I(i, t)-\langle I(i)\rangle] \times[I(j, t)-\langle I(j)\rangle]
$$

The resulting covariance map from the time range $37-72 \mathrm{~s}$ is shown in Figure 3b; additional time periods can be found in the Supporting Information in Figure S6b-d. Fluctuations in total Raman intensity, for example from changes in tip enhancement, give rise to an offset of all Raman active modes, appearing as correlated (positive value, blue) on the diagonal. In contrast, off-diagonal features are signatures of mode intensity correlations and anticorrelations. As can be seen, both correlated (blue) and anticorrelated (red) behavior can be observed between specific modes.

Figure $3 \mathrm{~d}$ shows the anticorrelation in the time domain in a subset of consecutive spectra from the time series data (indicated by the dashed area in Figure 3a). We find that vibrational modes of particular symmetries are suppressed in individual spectra. Adjacent peaks at 525 and $528 \mathrm{~cm}^{-1}$ and 799 and $804 \mathrm{~cm}^{-1}$, respectively, appear and disappear in an anticorrelated manner. Within the same time-series data, several other MG vibrational modes appear to remain constant in frequency and do not undergo blinking, including the mode at $580 \mathrm{~cm}^{-1}$. From the mode assignment based on DFT, we thus conclude that the appearance of modes of $A_{2}$ or $B_{1}$ symmetry are mutually exclusive and thus anticorrelated (see 46, 48, and $50 \mathrm{~s}$ spectra) except for rare transition events (spectra at 47 and $49 \mathrm{~s}$ ). In contrast, modes of identical symmetries are correlated, for example, the 525 and $799 \mathrm{~cm}^{-1}$ $B_{1}$ symmetry modes.

We model these time-dependent intensity fluctuations and spectral (anti) correlations through the tensor polarizability of surface-enhanced or tip-enhanced resonance Raman. Using selection rules developed for SERS, it is possible to assign molecular orientation. In resonance Raman, Frank-Condon selection rules allow observation of fully symmetric modes, while vibronic (Herzberg-Teller) coupling additionally allows observation of non-totally symmetric modes. As the optical frequency approaches a molecular electronic resonance, vibronically allowed modes can contribute significantly to the spectrum. $^{31-33}$ Near a metal surface, surface plasmon resonances further enhance the signal. ${ }^{34,35}$ Coupling between vibrations, molecular resonances and the metal substrate 
introduces more complex selection rules, which can be expressed as $\left\langle I\left|\mu^{\sigma}\right| K\right\rangle\left\langle K\left|\mu^{\rho}\right| F\right\rangle\left\langle F\left|V_{e N} / Q_{k}\right| I\right\rangle .^{32}$ Here $I, F$, and $K$ are the initial state, charge transfer state, and excited molecular state, respectively with associated dipole operators for the molecular $\mu^{\sigma}$, charge transfer $\mu^{\rho}$, and vibronic $V_{e N} / Q_{k}$ coupling. The molecular vibration, molecular electronic resonance and metal substrate thus each contribute to the observed intensity.

For a surface adsorbed molecule, the molecular symmetry is reduced and plasmonic enhancement of modes with particular symmetry can lead to surface selection rules based on molecular orientation..$^{32,36-39}$ Enhancement or suppression of vibronically enhanced modes in many cases can be used to determine molecular orientation on the surface. ${ }^{37,38,40,41}$ For aromatic molecules with $C_{2 v}$ symmetry such as MG (Figure 3c), $A_{2}$ modes are expected to be suppressed with the molecular $z$-axis normal to the surface, $B_{1}$ modes are suppressed with the $z$-axis parallel to the surface and aromatic plane normal to the surface, and $B_{2}$ modes are suppressed with the aromatic plane parallel to the surface, respectively.

Orthogonal vibrational modes are mutually exclusive within a single molecule or oriented cluster and necessarily appear anticorrelated with time-varying molecular orientation. Following the selection rules observed previously, ${ }^{37,41}$ we can assign anticorrelation between modes with $A_{2}$ and $B_{1}$ symmetries to rotational motion of an adsorbed MG molecule. Details of the coupling to both the surface plasmon and molecular electronic state at the excitation wavelengths may affect the orientational assignment of the molecule, though this is unlikely to affect orthogonality of Raman modes and the resulting anticorrelated fluctuations during rotation.

The frequency correlation, defined as the normalized covariance $\chi_{i j}=\sigma_{i j} /\left(\sigma_{i i} \sigma_{j j}\right)$, is shown in Supporting InformationFigure S6a. Fluctuations between modes with orthogonal polarizability exhibit a frequency correlation of -1 to -0.6 , while modes with the same symmetry have a correlation of +0.8 to +1 . This closely matches the idealized (anti) correlations of $(-1)$ and $+1 .^{42,43}$ Similarly, fully symmetric $\left(A_{1}\right)$ modes are positively correlated with all other modes. While small intensity fluctuations would also be possible in time series measurement of a tip wandering across small clusters of molecules, we observe much larger fluctuations. Individual modes in Figure 3d have intensities which fluctuate discretely between maximum and zero intensity, indicating single-molecule spectra are observed within the time-series.

Although enhancement by electronic coupling may affect the determination of the absolute orientation and angles of rotation, the mechanism of rotation based upon ground-state DFT calcuations is commensurate with our observations. Alternating enhancement between modes can occur as the molecule rotates in a pinwheel fashion with the aromatic plane tilted up from the surface and the phenyl moiety pointing either normal or parallel to the surface as illustrated in Figure 3e.

Additionally, from the studies of a larger time window, spectral fluctuations are observed in the form of frequency shifts. As an example, Figure 4a shows a selection of TERS spectra with short averaging times collected as a $209 \mathrm{~s}$ time series with a longer time series shown in Supporting Information Figure S4a. Individual TERS spectra have line widths significantly narrower than the far-field Raman spectrum, yet the sum of TERS spectra approaches the micro-Raman line shape, which averages over the spectral fluctuations and rotational motion occurring within the ensemble.
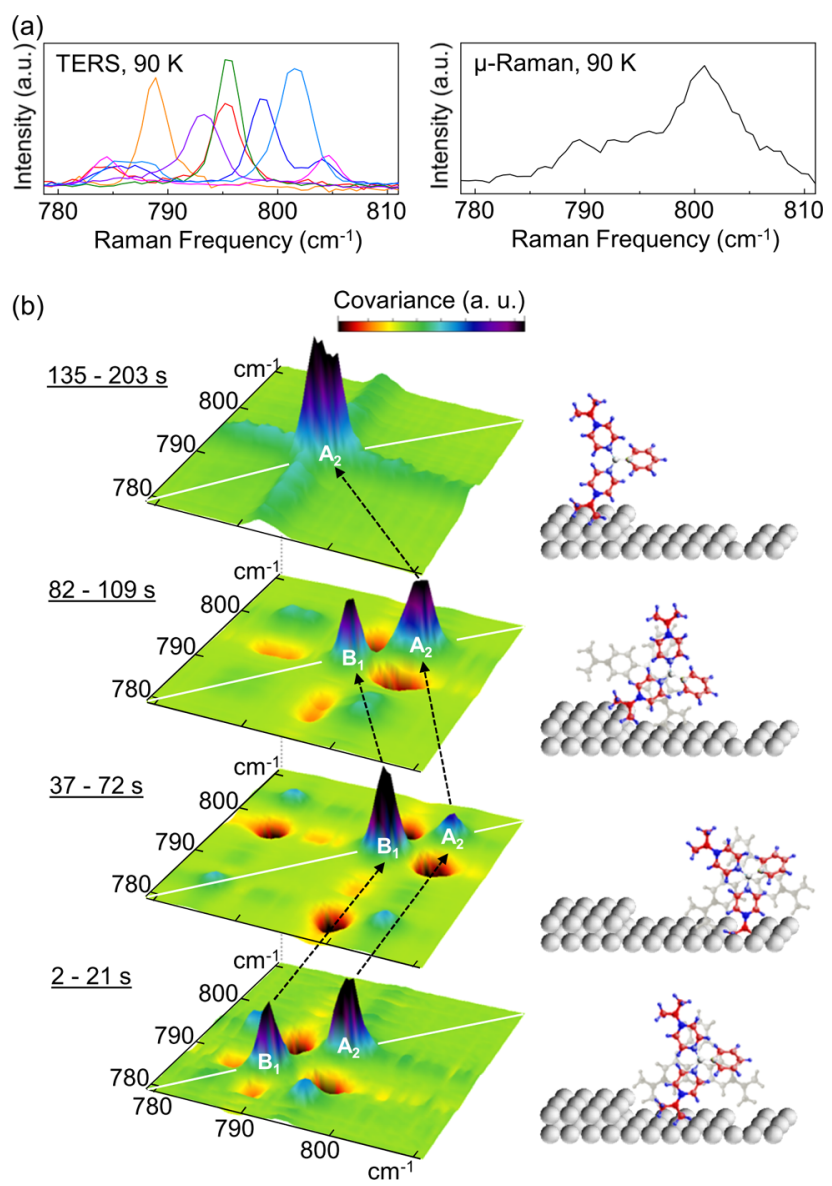

Figure 4. (a) Spectral fluctuations as observed in TERS spectra (left), compared with inhomogeneously broadened micro-Raman spectra (right), both at $90 \mathrm{~K}$. (b) Corresponding covariance plots with assigned underlying rotational motion within each of a series of short time segments. Spectral fluctuations appear as jumps in frequency between each time segment.

Figure $4 \mathrm{~b}$ shows covariance plots of the $780-810 \mathrm{~cm}^{-1}$ region for four short segments within the long $209 \mathrm{~s}$ time-series (see Supporting Information Figure S6b-d). Hydrogen out-ofplane bending modes with $A_{2}$ and $B_{1}$ symmetry show anticorrelated fluctuations during each of the first three time segments. In the final 135-203 s period, only a single mode is observed, and the molecule appears to remain stationary. Between the time segments, each of the hydrogen out-of-plane modes of $A_{2}$ and $B_{1}$ symmetry shift by a few $\mathrm{cm}^{-1}$. In contrast, during this same time period of $209 \mathrm{~s}$, several modes with fully symmetric $A_{1}$ character remain at nearly constant frequency throughout the acquisition and do not undergo blinking. We discuss below the covariance plots of short time segments and the correlation between modes with respect to dynamics of rotational motion and spectral fluctuations.

Discussion. In the following, we first discuss the temperature-dependent spectral narrowing and frequency shifts, followed by analysis of the rotational motion and spectral fluctuations. Temperature-activated spectral broadening and shifts are caused by ultrafast vibrational dephasing through intramolecular coupling to low-energy molecular modes. The correlated intensity fluctuations of particular vibrational modes directly record molecular reorientation occurring on the experimental time scale of seconds. 
Intramolecular Coupling. Temperature-dependent vibrational line widths range from $20 \mathrm{~cm}^{-1}$ for micro-Raman at 300 $\mathrm{K}$ to $1.5 \mathrm{~cm}^{-1}$ for TERS at $90 \mathrm{~K}$, in agreement with typical experimental measurements of linewidths in small molecules. $^{44,45}$ Similarly, extracted values for $\Gamma_{0}$ ranging 1.5 to 3.5 $\mathrm{cm}^{-1}$ compare well with typical values for molecular systems. ${ }^{46}$

The observed activated temperature dependence of both narrowing and frequency shifting of the peaks is characteristic of vibrational dephasing. Exchange modes mediating vibrational dephasing have been previously assigned to low energy intra- or inter-molecular vibrations, frustrated rotations, translations, or substrate phonons based upon the extracted $E_{\mathrm{A}}$ and the frequencies of available modes. ${ }^{21,30,47,48}$ The four vibrational modes analyzed in Table 1 exhibit values of $E_{\mathrm{A}}$ corresponding to specific frequencies ranging from 270 to $590 \mathrm{~cm}^{-1}$. A number of low energy torsions of $\mathrm{MG}$ are available near the observed $E_{\mathrm{A}}$, which can act as exchange modes, whereas phone modes of Au with energies limited to $\leq 160 \mathrm{~cm}-1$ are less likely to contribute. ${ }^{49}$ The extracted coupling strength $\delta \bar{\nu} \simeq 110 \mathrm{~cm}^{-1}$ falls within the range of values expected for intramolecular coupling, though it is somewhat larger than that observed in previous investigations of vibrational exchange $e^{26,28}$ or calculations of typical anharmonic coupling. ${ }^{50}$

Temperature-dependent TERS must be treated as an upper bound measurement of narrowing and dephasing by anharmonic coupling and exchange mode dephasing. Typically, temperature-dependent homogeneous broadening is measured in highly crystalline samples ${ }^{30}$ or using line-narrowing timedomain spectroscopies. ${ }^{51}$ Single-molecule vibrational spectroscopy has been proposed as an alternative measurement of the homogeneous line width, ${ }^{21,52}$ yet this assertion relies on the assumption of a stationary molecule within a well-defined local environment. TERS spectra of even a single molecule at room temperature are broadened due to rapid frequency fluctuations faster than the integration time of the measurement. For a small ensemble measurement, broadening at high temperatures contains contributions from both femtosecond vibrational dephasing and slower dynamics such that $E_{\mathrm{A}}$ and $A$ are overestimated by the exchange coupling model.

Within the spectral acquisition time a small ensemble or a single molecule can explore a large phase space and many local environments. At $90 \mathrm{~K}$, the molecular motion slows to a time scale less than the $1 \mathrm{~s}$ acquisition time, yet at $300 \mathrm{~K}$, the fluctuations occur too rapidly and contribute to line broadening. As seen in Figure $4 \mathrm{a}$, the series of $90 \mathrm{~K}$ TERS spectra collected with short acquisition times over the course of $209 \mathrm{~s}$ have narrow line widths comparable to the expected homogeneous line widths.

Single-Molecule Motion. With longer acquisition times or at higher temperature, individual acquisitions integrate over rapid spectral fluctuations, and broadening is observed similar to the spatial integration and ensemble broadening observed in farfield micro-Raman. We attribute these frequency fluctuations to two types of dynamic processes, rotation of the molecule relative to the surface and time-dependent changes in the local chemical environment, discussed below. Indeed, we only observe anticorrelated fluctuations on the $1 \mathrm{~s}$ time scales of spectral acquisition at $90 \mathrm{~K}$. At room temperature, molecular motion occurs too rapidly, and the acquisition averages over fluctuations such that the spectrum cannot be distinguished from an ensemble measurement.

Statistical analysis of fluctuating spectra through their covariance has been shown to sensitively isolate behavior of small subensembles within even bulk-type experiments. ${ }^{53}$ Anticorrelated fluctuations are often used as evidence for single-molecule spectral response in a number of singlemolecule methods. ${ }^{54}$ In TERS, fluctuations with intensity correlated between all modes have been often observed and attributed to variation in total enhancement. ${ }^{42}$ However, anticorrelated alternating Raman enhancement of one isotopologue relative to another was used to demonstrate singlemolecule response. ${ }^{55-57}$ Intensity fluctuations in isotopologues are attributed to enhancement of the full vibrational spectrum of one molecule relative to another resulting from diffusion of the tip, molecule, or localization of the field enhancement. Anticorrelated fluctuations in the TERS spectrum have also shown isomerization of a single photoswitching molecule on the apex of a TERS tip. ${ }^{58}$ Anticorrelated fluctuations in the time-series spectra are able to resolve single-molecule isomerization even within the larger probed ensemble of a selfassembled monolayer. Similar anticorrelated frequency fluctuations of certain modes in SERS of a lipid bilayer were attributed to single-molecule motion of a lipid within a membrane bilayer. $^{59}$

Rotational motion has been proposed to be observable through anticorrelated intensity fluctuations in modes with orthogonal polarizability tensors. ${ }^{42}$ Anticorrelated fluctuations have recently been observed in TERS, however these anticorrelations occurred between large several hundred $\mathrm{cm}^{-1}$ broad regions of the TERS spectrum, and were to slight variation in Frank-Condon enhancement and changes in the molecular electronic state. ${ }^{43}$ Near resonance with the molecular electronic state and near resonance with the tip plasmon, however, vibronic Herzberg-Teller enhancement can be larger than Franck-Condon enhancement, ${ }^{36,40,60}$ leading to our observed orientation-dependent Raman scattering from individual modes of different symmetry separated by only a few $\mathrm{cm}^{-1}$.

Spectral diffusion similarly slows to time scales that allow it to be observed as discrete frequency jumps in time series measurements at $90 \mathrm{~K}$. At several points within the time series, $A_{2}$ and $B_{1}$ modes jump coincidentally and with equal frequency shifts, while other modes remain at nearly constant frequency. Coincident shifts of the $A_{2}$ and $B_{1}$ modes are expected, because these normal modes all involve out-of-plane bending motions of the same set of $\mathrm{C}-\mathrm{H}$ bonds and differ only in symmetry. Further, changes in tip-sample geometry can affect the electronic and plasmonic coupling, this is expected to couple similarly to each of the observed modes and thus primarily affect the overall enhancement. ${ }^{43}$ Differences in vibrational frequency have been observed between subsequent singlemolecule SERS without time-resolved measurements, which was attributed to differences in a static local environment. ${ }^{24}$ Frequency wandering has also been observed in time-series SERS and TERS measurements, and observed motion was attributed to diffusion of liquid water. ${ }^{43,59}$ In vacuum at low temperature, however, frequency jumps are unlikely to be due to diffusion of residual liquid water and must instead be activated by the thermal bath. In contrast to the results obtained under atmospheric conditions, in vacuum we observe no fluctuations at room temperature and only minimal differences between repeated measurements at different locations. The disappearance of fluctuations with increasing temperature and associated increase in line width together support that frequency shifts at room temperature occur faster than the $1 \mathrm{~s}$ acquisition time and contribute to broadening. 
Analysis of mode character and fluctuation during a time series measurement supports assignments of rotational motion and spectral fluctuations over alternate explanations. Instability in the TERS probe or spectrometer can be ruled out because several torsional modes are stable to within $\leq 2 \mathrm{~cm}^{-1}$ over the same $209 \mathrm{~s}$ time-series (Supporting Information Figure S5b,c) during which the $\mathrm{C}-\mathrm{H}$ out-of-plane modes shift by several $\mathrm{cm}^{-1}$. If the tip was to drift, alternating enhancement of adjacent single molecules or oriented clusters should manifest as anticorrelated fluctuations similar to those expected for rotation of a single molecule. For the sparse coverages used in our experiment, however, sample drift or photobleaching at long times only causes the disappearance of all TERS signal, as sample motion and molecular diffusion are apparently insufficient to bring a new molecule into the TERS hotspot.

A remaining possibility is that charge transfer between the metal and molecule could affect both the chemical enhancement and shift the frequency of vibrational modes through the Stark effect. ${ }^{61}$ This is an unlikely explanation, however, as the tip and sample are held at ground potential. Discrete frequency switching is also possible in the case of rotation and diffusion of a small cluster in which all molecules move identically. Although we also consider this scenario unlikely, discriminating a single molecule from two molecules moving completely in tandem is a general limitation in single-molecule spectroscopy and that possibility cannot be completely eliminated. Drift of the tip or sample could also affect the angle of fieldenhancement and thus perceived molecular orientation, however, this is unlikely due to the stable tip-sample positions achieved at low temperatures.

The spectral fluctuations and rotational motion we observe approach the long-sought goal of measuring single-molecule local dynamics with structural sensitivity. Frequency shifts of vibrations are typically smaller than in fluorescence, yet vibrational modes are both structurally specific and typically more localized within a molecule, enabling direct and quantitative investigation of the chemical environment. For a molecule experiencing dynamic fluctuations within a heterogeneous local environment, rapid diffusion can result in a signal integrated over many molecular configurations and interactions with the environment, such that the signal of even a single molecule approaches that of an ensemble measurement. Selective detection of uncorrelated molecular motion is thus only possible in a highly controlled environment often requiring low temperatures.

Our demonstration of single-molecule dephasing, local dynamics, and rotational motion using TERS has not been previously possible in room temperature, ${ }^{17}$ low but constant temperature, and/or low spectral resolution experiments. ${ }^{19,20}$ Diffusion is slowed from fast room-temperature motions to the seconds time scale by cooling below the thermal activation energy, allowing for the observation of how the molecule dynamically samples the local energetic landscape. Because several of the anticorrelated modes are separated by $\leq 10 \mathrm{~cm}^{-1}$, even static orientation may be difficult to ascertain with other methods, for example, with lower spectral resolution, spatially averaged far-field Raman, or within the energetically disordered probe regions of SERS. Observation of intramolecular and conformational dynamics becomes possible through the combined high spectral resolution and single-molecule sensitivity of the experiment.

Conclusions. In summary, simultaneous access to disentangled intramolecular structure and dynamics, rotational motion, and spectral fluctuations has been a long-standing goal. Both single-molecule and ultrafast multi-dimensional spectroscopies have sought to separate and distinguish dynamic components from the heterogeneity of a system to investigate structural evolution and energy flow. Using variable temperature single-molecule TERS, we obtain detailed information about these molecular processes even from continuous wave experiments. The vibrational spectroscopy on the singlemolecule level with high spectral resolution, molecular fluctuations and their correlations on multiple time scales provide the desired detailed view of molecular motions. These explored fundamental molecular properties in a heterogeneous system are integral to the performance of a variety of functional materials and devices. High spectral resolution temperaturedependent TERS offers new routes for single-molecule investigations into intramolecular coupling, structure, vibrational dynamics, and molecular motion.

Methods. Figure 1a shows a schematic of our cryogenic TERS setup. A flow cryostat (ST 500, Janis) with vacuum chamber is pumped by a turbo-molecular pump to a base pressure of $<10^{-6} \mathrm{mbar}$. The sample is held by a cylindrical copper block (3 mm diameter) with K-type thermocouple. A coldfinger cools the sample to a minimum temperature of $90 \mathrm{~K}$ through an oxygen-free high conductivity (OFHC) copper braid connected to a liquid nitrogen cryostat. The temperature is controlled (Model 331 cryogenic temperature controller, Lake Shore Cryotronics) with a resistive heater and K-type thermocouple up to $300 \mathrm{~K}$ with a stability of better than $0.1 \mathrm{~K}$.

$\mathrm{Au}$ tips are etched electrochemically ${ }^{62}$ with $\sim 10 \mathrm{~nm}$ apex radius. A shear-force AFM based on a quartz tuning fork drives the tips at their resonance frequency $(\sim 32 \mathrm{kHz})$ with a dither amplitude of $<0.1 \mathrm{~nm} .{ }^{63}$ Amplitude of the tuning fork in shearforce contact with the sample provides feedback to maintain the tip at a constant tip-sample distance of $1-2 \mathrm{~nm} .{ }^{64}$ Sample drift for an equilibrated sample at $90 \mathrm{~K}$ is observed to be $<1 \mathrm{~nm} /$ min. The sample holder is mounted to a glass plate that is attached to a piezoelectric transducer (PZT, Attocube) for $x y z$ sample scanning. AFM operation and tip positioning are controlled by a digital AFM controller (R9, RHK Technology) with a stepper motor (MX25, Mechonics) for coarse positioning.

The tip and sample are positioned in the center of a custom $25 \mathrm{~mm}$ parabolic mirror (PM), Ag-coated, focal length $=12$ $\mathrm{mm}$, and NA = 1.0. ${ }^{65,66}$ A helium-neon laser $(632.8 \mathrm{~nm}, P<$ $0.3 \mathrm{~mW})$ and liquid-crystal based polarization converter (ARCoptix) provide a radial polarized incident beam ${ }^{67}$ aligned with respect to the PM and focused onto the tip apex with a measured spot size of radius $1 \mu \mathrm{m}$.

At the excitation wavelength of $632.8 \mathrm{~nm}(1.96 \mathrm{eV}) \mathrm{MG}$ undergoes a resonance Raman scattering through an electronic transition of the $\pi$-conjugated backbone. The tip-sample coupling is enhanced through localized dipole-dipole interaction between plasmonic tip and metallic substrate. ${ }^{15}$ Raman signal is collected in the back scattered direction, passed through a long-pass filter (633 nm cutoff) and focused onto the slit of a spectrometer $(\mathrm{f}=500 \mathrm{~mm}$, SpectraPro 500i, Princeton Instruments) with a $N_{2}(l)$ cooled charge-coupled device (CCD, Spec-10 LN/100BR, Princeton Instruments). Far-field microRaman is measured using the same experimental setup with the AFM tip retracted several hundred $\mu \mathrm{m}$ from the sample. The spectrometer is calibrated using hydrogen and mercury lines with measured spectral resolution of $1.2 \mathrm{~cm}^{-1}$ using a 1200 groove/mm grating. Experimental assignments of vibrational 
modes are made by comparison with DFT calculations using Gaussian98 at the RB3LYP $\backslash 6-31 G(d, p)$ level.

TERS measurements are performed in a three step procedure. First, laser beam and sample are positioned and aligned for optimal focus on the sample surface. Second, after retracting the sample, the tip is moved into the focus of the laser beam. Last, the sample is brought into shear-force AFM feedback for TERS measurements.

The MG sample is prepared by spin coating from an ethanol solution on a $100 \mathrm{~nm}$ thick Si-template stripped Au layer (surface $\mathrm{rms}$ roughness $<0.5 \mathrm{~nm}$ ) at a rate of $3000 \mathrm{rpm}$ for 2 min. Coverage is calibrated by measuring the visible absorption (Cary 500 Scan UV-vis-NIR spectrophotometer) of reference samples spin-coated onto glass and calculating coverage from the known optical cross section of MG and monolayer density of 1 molecule $\mathrm{nm}^{-2}$ (see Supporting Information Figure S1a). For the far-field micro-Raman reference measurements, $\sim 0.14$ monolayer (ML) sample coverage is used and for the TERS measurements we use $\sim 0.006 \mathrm{ML}$ coverage. On the basis of this calculation, we probe $4 \times 10^{5} \mathrm{MG}$ molecules within the measured micro-Raman spot size of radius $1 \mu \mathrm{m}$. In contrast, an AFM tip with radius $10 \mathrm{~nm}$ probes an average of $\sim 1-2$ molecules in TERS, enabling routine few-molecule subensemble measurements. TERS measurements confirm a similarly low surface coverage. TERS signal only occurs at sparsely distributed sample locations, corresponding to less than $10 \%$ of locations at $90 \mathrm{~K}$. Assuming molecules are distributed individually or sometimes clustered, single-molecule spectra are possible at this coverage, and approximately a third of spectra at $90 \mathrm{~K}$ show the fluctuations associated with singlemolecule response in time-series measurements.

\section{ASSOCIATED CONTENT}

\section{S Supporting Information}

The Supporting Information is available free of charge on the ACS Publications website at DOI: 10.1021/acs.nanolett.5b04135.

Experimental procedures and characterization data for malachite green samples. Additional data is included in support of the temperature-dependent spectral features and time-series fluctuations. (PDF)

\section{AUTHOR INFORMATION}

\section{Corresponding Author}

*E-mail: markus.raschke@colorado.edu.

\section{Author Contributions}

K.-D.P. and E.A.M.contributed equally to this work.

\section{Notes}

The authors declare no competing financial interest.

\section{ACKNOWLEDGMENTS}

The authors would like to thank Molly May for contributions to instrument development. We acknowledge funding from the Department of Energy Division of Materials Sciences and Engineering (Grant DE-SC0008807), and the National Science Foundation (NSF Grant CHE1306398).

\section{REFERENCES}

(1) Moerner, W.; Kador, L. Phys. Rev. Lett. 1989, 62, 2535-2538.

(2) Hell, S. W.; Wichmann, J. Opt. Lett. 1994, 19, 780.
(3) Betzig, E.; Patterson, G. H.; Sougrat, R.; Lindwasser, O. W.; Olenych, S.; Bonifacino, J. S.; Davidson, M. W.; Lippincott-Schwartz, J.; Hess, H. F. Science 2006, 313, 1642-5.

(4) Min, W.; Lu, S.; Chong, S.; Roy, R.; Holtom, G. R.; Xie, X. S. Nature 2009, 461, 1105-9.

(5) Bout, D. A. V.; Yip, W.-T.; Hu, D.; Fu, D.-K.; Swager, T. M.; Barbara, P. F. Science 1997, 277, 1074-1077.

(6) Tian, Y.; Navarro, P.; Kozankiewicz, B.; Orrit, M. ChemPhysChem 2012, 13, 3510-5.

(7) Sosa, H.; Peterman, E. J.; Moerner, W. E.; Goldstein, L. S. Nat. Struct. Biol. 2001, 8, 540-4.

(8) Pertsinidis, A.; Zhang, Y.; Chu, S. Nature 2010, 466, 647-651.

(9) Goldsmith, R. H.; Moerner, W. E. Nat. Chem. 2010, 2, 179-86.

(10) Sallen, G.; Tribu, A.; Aichele, T.; André, R.; Besombes, L.; Bougerol, C.; Richard, M.; Tatarenko, S.; Kheng, K.; Poizat, J.-P. Nat. Photonics 2010, 4, 696-699.

(11) Rosenfeld, D. E.; Gengeliczki, Z.; Smith, B. J.; Stack, T. D. P.; Fayer, M. D. Science 2011, 334, 634-9.

(12) Kneipp, K.; Wang, Y.; Kneipp, H.; Perelman, L.; Itzkan, I.; Dasari, R.; Feld, M. Phys. Rev. Lett. 1997, 78, 1667-1670.

(13) Nie, S. Science 1997, 275, 1102-1106.

(14) Stöckle, R. M.; Suh, Y. D.; Deckert, V.; Zenobi, R. Chem. Phys. Lett. 2000, 318, 131-136.

(15) Neacsu, C. C.; Dreyer, J.; Behr, N.; Raschke, M. B. Phys. Rev. B: Condens. Matter Mater. Phys. 2006, 73, 193406.

(16) Stadler, J.; Schmid, T.; Zenobi, R. Nano Lett. 2010, 10, 451420.

(17) van Schrojenstein Lantman, E. M.; Deckert-Gaudig, T.; Mank, A. J. G.; Deckert, V.; Weckhuysen, B. M. Nat. Nanotechnol. 2012, 7, $583-6$.

(18) Zhang, W.; Yeo, B.; Schmid, T.; Zenobi, R. J. Phys. Chem. C 2007, 111, 1733-1738.

(19) Zhang, R.; Zhang, Y.; Dong, Z. C.; Jiang, S.; Zhang, C.; Chen, L. G.; Zhang, L.; Liao, Y.; Aizpurua, J.; Luo, Y.; Yang, J. L.; Hou, J. G. Nature 2013, 498, 82-6.

(20) Klingsporn, J. M.; Jiang, N.; Pozzi, E. A.; Sonntag, M. D.; Chulhai, D.; Seideman, T.; Jensen, L.; Hersam, M. C.; Van Duyne, R. P. J. Am. Chem. Soc. 2014, 136, 3881-7.

(21) Artur, C.; Le Ru, E. C.; Etchegoin, P. G. J. Phys. Chem. Lett. 2011, 2, 3002-3005.

(22) Pettinger, B.; Ren, B.; Picardi, G.; Schuster, R.; Ertl, G. Phys. Rev. Lett. 2004, 92, 096101.

(23) Yampolsky, S.; Fishman, D. A.; Dey, S.; Hulkko, E.; Banik, M.; Potma, E. O.; Apkarian, V. A. Nat. Photonics 2014, 8, 650-656.

(24) Etchegoin, P. G.; Le Ru, E. C. Anal. Chem. 2010, 82, 2888-92.

(25) Redfield, A. G. IBM J. Res. Dev. 1957, 1, 19-31.

(26) Harris, C.; Shelby, R.; Cornelius, P. Phys. Rev. Lett. 1977, 38, 1415-1419.

(27) Shelby, R. M.; Harris, C. B.; Cornelius, P. A. J. Chem. Phys. 1979, 70, 34

(28) Persson, B.; Ryberg, R. Phys. Rev. B: Condens. Matter Mater. Phys. 1985, 32, 3586-3596.

(29) Skinner, J. L.; Hsu, D. J. Phys. Chem. 1986, 90, 4931-4938.

(30) Karavitis, M.; Kumada, T.; Goldschleger, I. U.; Apkarian, V. A. Phys. Chem. Chem. Phys. 2005, 7, 791.

(31) Albrecht, A. C. J. Chem. Phys. 1961, 34, 1476.

(32) Lombardi, J. R.; Birke, R. L. Acc. Chem. Res. 2009, 42, 734-742.

(33) Baiardi, A.; Bloino, J.; Barone, V. J. Chem. Phys. 2014, 141, 114108 .

(34) Banik, M.; El-Khoury, P. Z.; Nag, A.; Rodriguez-Perez, A.; Guarrottxena, N.; Bazan, G. C.; Apkarian, V. A. ACS Nano 2012, 6, $10343-54$.

(35) El-Khoury, P. Z.; Hu, D.; Hess, W. P. J. Phys. Chem. Lett. 2013, 4, 3435-3439.

(36) Moskovits, M. J. Chem. Phys. 1982, 77, 4408.

(37) Creighton, J. Surf. Sci. 1983, 124, 209-219.

(38) Hildebrandt, P.; Stockburger, M. J. Phys. Chem. 1984, 88, 59355944.

(39) Campion, A.; Kambhampati, P. Chem. Soc. Rev. 1998, 27, 241. 
(40) Osawa, M.; Matsuda, N.; Yoshii, K.; Uchida, I. J. Phys. Chem.

1994, 98, 12702-12707.

(41) Chaigneau, M.; Picardi, G.; Ossikovski, R. Int. J. Mol. Sci. 2011, $12,1245-58$.

(42) Moore, A. A.; Jacobson, M. L.; Belabas, N.; Rowlen, K. L.; Jonas, D. M. J. Am. Chem. Soc. 2005, 127, 7292-7293.

(43) Sonntag, M. D.; Chulhai, D.; Seideman, T.; Jensen, L.; Van Duyne, R. P. J. Am. Chem. Soc. 2013, 135, 17187-17192.

(44) Surovtsev, N. V.; Malinovsky, V. K.; Boldyreva, E. V. J. Chem. Phys. 2011, 134, 045102.

(45) Kim, Y. S.; Hochstrasser, R. M. J. Phys. Chem. B 2009, 113, 8231-51.

(46) Rey, R.; Møller, K. B.; Hynes, J. T. Chem. Rev. 2004, 104, 191528.

(47) Moritsugu, K.; Miyashita, O.; Kidera, A. J. Phys. Chem. B 2003, 107, 3309-3317.

(48) Vojta, D.; Baranović, G. Vib. Spectrosc. 2010, 52, 178-187.

(49) Bian, Q.; Bose, S.; Shukla, R. J. Phys. Chem. Solids 2008, 69, $168-181$.

(50) Kasyanenko, V. M.; Keiffer, P.; Rubtsov, I. V. J. Chem. Phys. 2012, 136, 144503.

(51) Rector, K. D.; Fayer, M. D. Int. Rev. Phys. Chem. 1998, 17, 261306.

(52) Hoggard, A.; Wang, L.-Y.; Ma, L.; Fang, Y.; You, G.; Olson, J.; Liu, Z.; Chang, W.-S.; Ajayan, P. M.; Link, S. ACS Nano 2013, 7, 11209-11217.

(53) Streit, J. K.; Bachilo, S. M.; Sanchez, S. R.; Lin, C.-W.; Weisman, R. B. J. Phys. Chem. Lett. 2015, 6, 3976-3981.

(54) Ha, T.; Enderle, T.; Ogletree, D. F.; Chemla, D. S.; Selvin, P. R.; Weiss, S. Proc. Natl. Acad. Sci. U. S. A. 1996, 93, 6264-6268.

(55) Kleinman, S. L.; Ringe, E.; Valley, N.; Wustholz, K. L.; Phillips, E.; Scheidt, K. A.; Schatz, G. C.; Van Duyne, R. P. J. Am. Chem. Soc. 2011, 133, 4115-22.

(56) Dieringer, J. A.; Lettan, R. B.; Scheidt, K. A.; Van Duyne, R. P. J. Am. Chem. Soc. 2007, 129, 16249-56.

(57) Sonntag, M. D.; Klingsporn, J. M.; Garibay, L. K.; Roberts, J. M.; Dieringer, J. A.; Seideman, T.; Scheidt, K. A.; Jensen, L.; Schatz, G. C.; Van Duyne, R. P. J. Phys. Chem. C 2012, 116, 478-483.

(58) Tallarida, N.; Rios, L.; Apkarian, V. A.; Lee, J. Nano Lett. 2015, $15,6386$.

(59) Taylor, R. W.; Benz, F.; Sigle, D. O.; Bowman, R. W.; Bao, P.; Roth, J. S.; Heath, G. R.; Evans, S. D.; Baumberg, J. J. Sci. Rep. 2014, 4, 5940.

(60) Gao, X.; Davies, J. P.; Weaver, M. J. J. Phys. Chem. 1990, 94, 6858-6864.

(61) Mirjani, F.; Thijssen, J. M.; Ratner, M. A. J. Phys. Chem. C 2012, 116, 23120-23129.

(62) Neacsu, C. C.; Steudle, G. A.; Raschke, M. B. Appl. Phys. B: Lasers Opt. 2005, 80, 295-300.

(63) Karrai, K.; Grober, R. D. Ultramicroscopy 1995, 61, 197-205. Selected Papers from the third International Conference on Near-Field Optics and Related Techniques.

(64) Kravtsov, V.; Berweger, S.; Atkin, J. M.; Raschke, M. B. Nano Lett. 2014, 14, 5270-5275.

(65) Sackrow, M.; Stanciu, C.; Lieb, M. A.; Meixner, A. J. ChemPhysChem 2008, 9, 316-20.

(66) Zhang, D.; Wang, X.; Braun, K.; Egelhaaf, H.-J.; Fleischer, M.; Hennemann, L.; Hintz, H.; Stanciu, C.; Brabec, C. J.; Kern, D. P.; Meixner, A. J. J. Raman Spectrosc. 2009, 40, 1371-1376.

(67) Kihm, H. W.; Kim, J.; Koo, S.; Ahn, J.; Ahn, K.; Lee, K.; Park, N.; Kim, D.-S. Opt. Express 2013, 21, 5625-33. 